\title{
LA “TÉCNICA DE CLARIFICACIÓN 5-5-5", UN MÉTODO NATURAL PARA EL TRATAMIENTO DE MATERIAL VEGETAL
}

\begin{abstract}
ANA M. ARAMBARRI ${ }^{1}$
Summary: The "clearing technique $5-5-5$ ", a natural method for treatment of plant materials. The objective of this paper is to give information on one alternative simple and economic clearing method, without aggressiveness for the material, which may be used for the investigation of different plant organs and tissues. Some years ago, a clearing technique of plant leaves began to test in the Laboratory of Comparative Morphology of Spermatophytes (LAMCE). It was developed in the search for a clearing method that would allow to us to maintain orientation of the material and was less aggressive for the small and delicate materials. As result a natural method with those characteristics was found.
\end{abstract}

Key words: Botanical technique, transparent plant materials.

\begin{abstract}
Resumen: El objetivo de esta publicación es dar a conocer, como alternativa, una técnica de clarificación, sencilla, económica, sin agresividad para el material vegetal y que puede ser empleada para la investigación de diferentes órganos y tejidos. Hace algunos años, se comenzó a ensayar en el Laboratorio de Morfología Comparada de Espermatófitas (LAMCE), una técnica de diafanización de hojas de plantas. La misma se desarrolló ante la búsqueda de un método de clarificación que permitiera mantener la orientación del material y no fuera tan agresivo para los materiales pequeños y delicados. Como resultado se obtuvo un método natural con las características buscadas.
\end{abstract}

Palabras clave: Material transparente, técnica botánica.

\section{INTRODUCCIÓN}

La hoja es el órgano de las plantas más sensible a los cambios ambientales. El tejido epidérmico es de interés en el campo de las plantas medicinales, en especial en la detección y control de calidad farmacobotánica (Altamirano \& Yajía, 2017) evitando adulteraciones, para lo cual se requieren herramientas rápidas y prácticas (Gattuso, 2013). Su estudio también es de valor, por ejemplo para el reconocimiento de plantas tóxicas para el ganado (Freire et al., 2005), en la determinación de la composición botánica de la dieta de herbívoros (Yagueddú \& Cid, 1992; Castellaro et al., 2007); Borelli \& Pelliza de Sbriller, 2014); en estudios de contenido estomacal resistente al proceso digestivo (Cid \& Sierra, 2004), en estudios de taxonomía (e. g. utilizando los índices estomático y piloso), ecofisiológicos utilizando densidad y tamaño de estomas-ostíolo e incluso en trabajos destinados a la conservación de especies.

La diafanización u obtención de material transparente (clarificación), es una técnica que permite estudiar el tejido epidérmico y observar características de los tejidos internos, por ejemplo características celulares del parénquima, las venas y vénulas (haces vasculares), aréolas, tamaño de espacios intercelulares, presencia y tipos de esclereidas, presencia de estructuras de secreción como tubos laticíferos e inclusiones celulares como los cristales. Además una buena clarificación es una herramienta rápida que le permite al investigador formar una imagen tridimensional del órgano.

\footnotetext{
1 Laboratorio de Morfología Comparada de Espermatófitas (LAMCE), Facultad de Ciencias Agrarias y Forestales Universidad Nacional de La Plata, Avda. 60 y 119, C.C. 31 (1900) La Plata, Argentina. Te. 54-0221-423-6758 ext. 461. anaramba@yahoo.com.ar; arambarri@agro.unlp.edu.ar
} 
Algunos de los numerosos protocolos que existen para la clarificación de materiales son: Johansen (1940), Foster (1949), Payne (1969), Herr, Jr. (1971), Dizeo de Strittmatter (1973), Vasco et al. (2014). En la mayoría de los métodos utilizan alcohol etílico que elimina la clorofila y otros contenidos celulares facilitando la clarificación; hidróxido de sodio que ablanda e hidrata el material; hipoclorito de sodio que también elimina contenidos celulares y hace transparente el material y el hidrato de cloral que actúa sobre los tejidos dejando translúcida la muestra. A veces, con esta finalidad se emplea el ácido láctico solo o conjuntamente con el hidrato de cloral (Herr, Jr., 1971).

Una de las técnicas de diafanización más utilizadas es la de Dizeo de Strittmatter (1973) con excelentes resultados. Sin embargo, la técnica exige ciertos cuidados durante y luego del proceso de ebullición del material (Zarlavsky, 2014), además si el material vegetal es delicado puede destruirse y no permite mantener orientado el material de estudio. Estos problemas hicieron que, cuando hubo que analizar ambas caras de los pequeños folíolos de segundo orden (foliólulos) de la hoja bipinnada de Acacia aroma Hook. \& Arn., surgió la idea de ensayar un método con el cual se pudiera mantener la orientación del material a lo largo de todo el proceso y diera un resultado satisfactorio desde el punto de vista de la transparencia.

\section{Materiales y Métodos}

\section{Materiales vegetales ensayados}

Este método natural se comenzó a utilizar en 2010, desde entonces se ha empleado en el estudio de plantas medicinales, alternando con la técnica de Dizeo de Strittmatter (1973), dependiendo del material, la elección de uno u otro método. Usamos ambas técnicas en los trabajos de especies arbóreas de la región chaqueña (Arambarri et al., 2011, 2012). La técnica de clarificación 5-5-5 permitió diafanizar las hojas de Celtis ehrenbergiana (Klotzsch) Liebm. (Nughes et al., 2013; Hernández et al., 2016) y observar tricomas gemelos completos, incluso con su contenido de mucílagos (e. g. Senecio laetevirens Phil.), en material de herbario de más de 50 años (comunicación personal M. P. Hernández).

Otros materiales ensayados fueron: hojas de Capsicum annuum L., Eryngium pandanifolium Cham. \& Schltdl., Hydrocotyle ranunculoides
L.f., Ipomoea cairica (L.) Sweet, Lactuca sativa L., Muehlenbeckia sagittifolia (Ortega) Meisn., Passiflora caerulea L., Salpichroa origanifolia (Lam.) Thell., Tropaeolum majus L; cladodios (segmentos de tallo) de Opuntia spp.; raicillas de Lycopersicon esculentum Mill.; flores de Eupatorium sp., Lantana camara L., Thunbergia alata Bojer ex Sims, Wedelia glauca (Ortega) O. Hoffm. ex Hicken; frutos (pericarpo) de Solanum melongena L.; semillas (episperma) de Datura ferox L. y D. stramonium L.; fruto y semilla inmaduros de Wedelia glauca; explantes de Prosopis alpataco Phil. (cultivo in vitro).

\section{"Técnica de clarificación 5-5-5"}

\section{Tratamiento previo a la clarificación}

$\mathrm{Si}$ bien generalmente cuando se presenta una técnica no se habla sobre el tratamiento previo del material, es un factor importante para el buen desarrollo de la misma. Se ha observado que los materiales presentan mayor o menor grado de dificultad para su clarificación. Se pueden enumerar los siguientes casos: (1) generalmente el material fresco es más difícil de diafanizar que el material de herbario, aunque en ambos casos depende del órgano y composición química del mismo; (2) el material de herbario requiere hidratación previa; (3) el comportamiento del material es distinto al fijarlo en solución de formalina-ácido acético glacial-alcohol (FAA). En algunos casos sufren al menos cierta decoloración y luego se diafanizan rápidamente. También están aquellos que colocados en FAA mantienen una coloración verde intensa e incluso se oscurecen; estos materiales se clarifican mal o lo hacen lentamente.

Vasco et al. (2014) observaron lo mismo en el material conservado en FAA y explicaron que la formalina del FAA puede fijar contenidos celulares (e. g. pigmentos, fenoles), que dificultan la clarificación del material, para reducir ese efecto indicaron dejar el material dos semanas en alcohol $50^{\circ}$. En el presente trabajo se ensayó el tratamiento con alcohol etílico $50^{\circ}$ con tres materiales de hojas que estuvieron desde uno hasta 10 años en el líquido fijador y mantuvieron el color verde intenso o se oscurecieron.

Los resultados comparativos entre el tratamiento de clarificación desde el material en FAA y luego del tratamiento durante 15 días en alcohol $50^{\circ}$ se presentan en la Tabla 1 y de ello surge la siguiente recomendación: 


\section{A. M. Arambarri - Nuevo método de clarificación vegetal}

-Si el material es fresco o de herbario hidratado y se lo va a clarificar dentro de las tres semanas, conviene conservarlo en alcohol $50^{\circ}$ por lo menos 15 días y luego iniciar el proceso de clarificación.

-Cuando el material está conservado en FAA y presenta coloración intensa, se recomienda colocarlo 15-20 días en alcohol $50^{\circ}$ antes de la clarificación. El material ensayado muestra que el tiempo total de clarificación con el tratamiento con alcohol $50^{\circ}$ fue mayor que si no se hace este tratamiento (Tabla 1). Sin embargo, se pueden citar como ventajas: 1) que puede lograrse una mejor clarificación; 2) el material queda expuesto durante menos tiempo a la solución de hidróxido de sodio+hipoclorito de sodio que pueden provocar un excesivo ablandamiento y rotura de sus partes.

\section{Clarificación \\ Reactivos utilizados: \\ -Hidróxido de sodio $(\mathrm{NaOH})$ al 5\% \\ -Hipoclorito de sodio ( $\mathrm{NaClO}$ ) al $5 \%$ y $50 \%$ \\ -Hidrato de cloral $\left(\mathrm{C}_{2} \mathrm{H}_{3} \mathrm{Cl}_{3} \mathrm{O}_{2}\right)$ al $5 \%$}

\section{Instrumental mínimo}

Potes de vidrio con tapa plástica, de $5 \mathrm{~cm}$ diámetro y profundidad interna de $3 \mathrm{~cm}$ como mínimo (con mayor diámetro facilita la tarea). Los recipientes deben ser de vidrio y la tapa del mismo material o de plástico para evitar la acción corrosiva de los reactivos.

Cajas de Petri de vidrio, de $10 \mathrm{~cm}$ de diámetro.

Pinzas de punta recta, las de punta plana facilitan asir el material.

Guantes de látex.

\section{Procedimiento}

En el pote de vidrio se vierten los reactivos hidróxido de sodio (5\%) e hipoclorito de sodio $(5 \%)$ en partes iguales (1: 1). Con una pinza se toma el material en la posición deseada y extrae de la solución de alcohol $50^{\circ}$ o FAA (Fig. 1A), se sostiene pasándolo por agua para lavarlo (Fig. 1B), luego se sumerge en la solución de reactivos (Fig. 1C); puede ser la hoja completa o porción media de la misma, con la cara adaxial hacia arriba y abaxial hacia abajo. El recipiente tapado, rotulado y sin agitar se mantiene a temperatura ambiente (Fig. 1D), realizando el control visual de la decoloración del material, lo cual ocurre en un tiempo variable desde pocos minutos hasta 10-15 días, en general son 4-5 días (Tabla 1). Cuando se ha decolorado, al menos parcialmente, ya que con frecuencia la vena media de la lámina demora más tiempo en decolorarse, con la pinza se toma la muestra manteniendo la posición, se extrae del recipiente y se somete al lavado (Fig. 1E). Este se hace en cajas de Petri, por la comodidad de su amplitud y poca profundidad ya que conviene no soltar el material, para evitar que se mueva y gire. El lavado se hace mínimo dos veces, en agua común o agua destilada ya que luego se lo pasa a otra caja de Petri conteniendo hipoclorito de sodio (50\%) donde se completa la decoloración, generalmente en unos segundos o minutos. El material totalmente decolorado se lava 3-5 veces, en cajas de Petri, por lo menos los tres últimos lavados con agua destilada, manteniendo siempre la posición, lo cual se puede hacer sin soltar la muestra (Fig. 1E). Una vez lavado, se continúa la marcha sumergiéndolo en una solución de hidrato de cloral (5\%) que se ha vertido en otra caja de Petri que se tapa, rotula y deja como mínimo 24 horas (Fig. 1F). En caso de obtener una total decoloración en la mezcla inicial de $\mathrm{NaOH}(5 \%)+\mathrm{NaClO}(5 \%)$, se salteará el paso de la decoloración con hipoclorito de sodio (50\%) y continuará con el lavado y sumersión en hidrato de cloral para su clarificación. Recordar que en el hidrato de cloral, el material puede permanecer varios días (nunca menos de un día).

\section{Coloración y montaje}

-Safranina al 1\% (1 g de safranina en $100 \mathrm{~mL}$ de agua destilada).

-Safranina a saturación en alcohol $80^{\circ}$.

- Fucsina básica $0,5 \%(0,5 \mathrm{~g}$ en $100 \mathrm{~mL}$ de alcohol $\left.50^{\circ}\right)$.

Para la coloración con safranina se puede seguir la técnica indicada por Dizeo de Strittmatter (1973) o realizar la coloración simple con safranina (Zarlavsky, 2014). También se puede preparar Gelatina-safranina y directamente montar en dicha solución, para ello se extrae del hidrato de cloral, se coloca en caja de Petri con agua destilada, desde donde se toma para colocar sobre el medio de montaje (Fig. 1G, H) y luego se desliza el cubreobjetos (Fig. 1I). Cuando el material es muy delicado como pétalos de la flor o una hoja bipinnada, se introduce el portaobjeto en el agua y se arrastra el material sobre el portaobjeto (Fig. 1J), luego se seca el exceso de agua, agrega gelatinasafranina y desliza el cubreobjeto. 
Bol. Soc. Argent. Bot. 53 (4) 2018
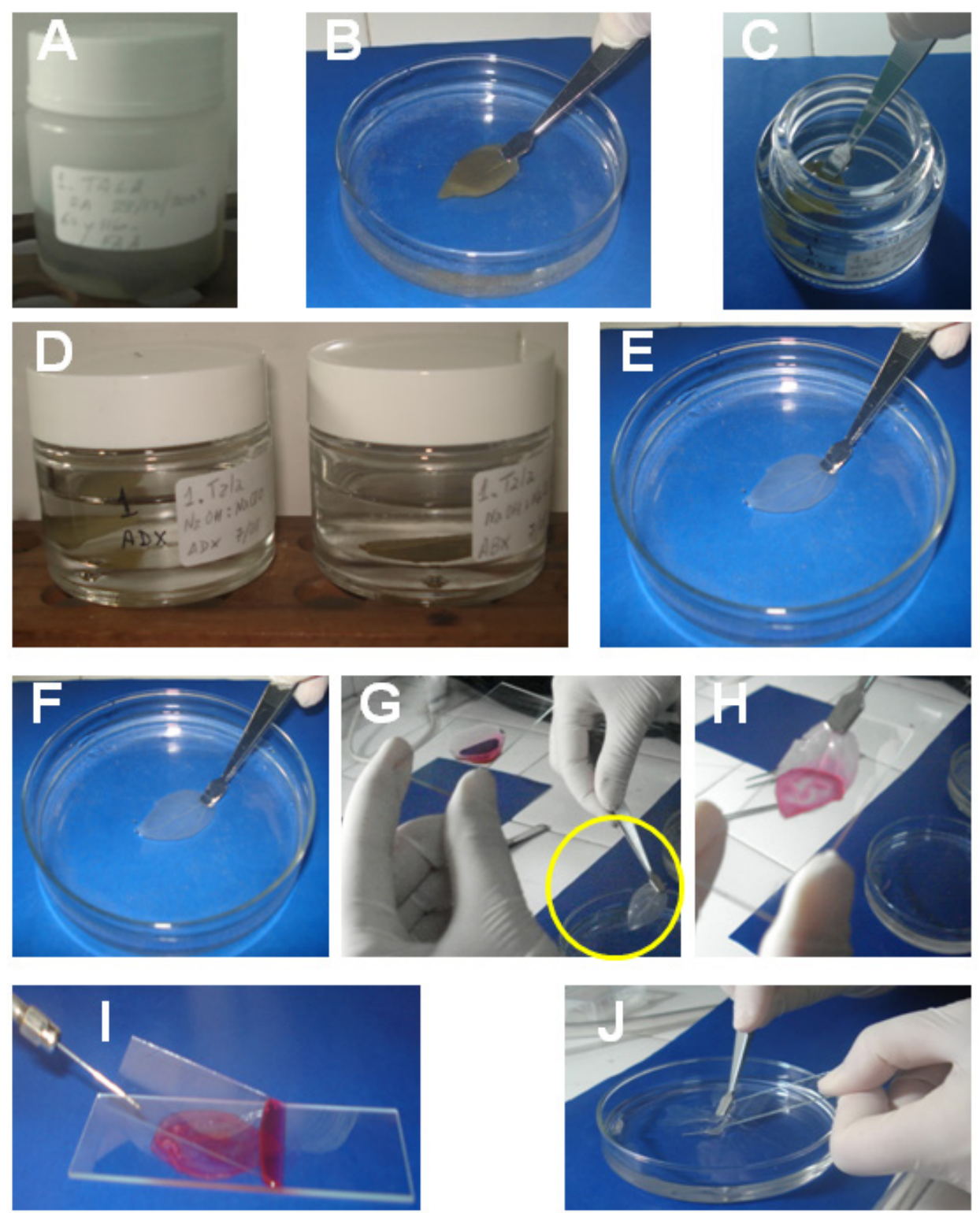

Fig. 1. Metodología. A: recipiente conteniendo el material en FAA. B: el material que es tomado con la pinza, se lava previo a colocarlo en el pote con la mezcla 1: 1 de hidróxido de sodio (5\%) e hipoclorito de sodio (5\%). C: colocación del material en la mezcla mencionada en un pote de no menos de $5 \mathrm{~cm}$ de diámetro. D: colocación (sin agitar) del pote cerrado y rotulado, sobre un soporte a temperatura ambiente, en el cual se controlará visualmente la decoloración. E: extracción con la pinza del material, decolorado, guardando siempre la posición, se hacen los lavados sin soltar el material. F: una vez totalmente decolorado se introduce en la misma posición en un recipiente con hidrato de cloral $(5 \%)$, mínimo 24 horas para su clarificación. G y H: con la pinza se toma el material del hidrato de cloral y se coloca en agua. Para el montaje se prepara el portaobjeto con la gelatina-safranina y con la pinza guardando la posición se toma el material del agua -señalado con un círculo sobre la foto- y coloca en el medio de montaje. I: se cubre deslizando el cubreobjeto. J: cuando la muestra es de un material muy delicado (pétalo de la flor u hoja bipinnada) se introduce el portaobjeto en el agua y arrastra el material extendido sobre el portaobjeto, luego se seca el excedente líquido, se agrega el medio de montaje y procede a cubrir con el cubreobjeto (como se indica en I). 
Tabla 1. Tiempos de clarificación con la técnica 5-5-5. Comparación de materiales vegetales conservados en FAA de diferentes especies seleccionadas. Sin tratamiento previo y con tratamiento previo de sumersión en alcohol etílico $50^{\circ}$ durante 15 días.

\begin{tabular}{|c|c|c|}
\hline Órganos y especies analizadas & $\begin{array}{c}\text { Sin tratamiento previo } \\
\text { con alcohol } 50^{\circ}\end{array}$ & $\begin{array}{l}\text { Con tratamiento previo } \\
15 \text { días con alcohol } 50^{\circ}\end{array}$ \\
\hline \multicolumn{3}{|l|}{ HOJAS } \\
\hline Acacia aroma & 4-5 días & \\
\hline Anadenanthera colubrina var. cebil & 4-5 días & \\
\hline Capsicum annuum & 4-5 días & \\
\hline Celtis ehrenbergiana & 4-15 días & 3-8 días \\
\hline Eryngium pandanifolium & 10-15 días & 3-4 días \\
\hline Hydrocotyle ranunculoides & 2-3 días & \\
\hline Muehlenbeckia sagittifolia & 8-12 días & 2-3 días \\
\hline Passiflora caerulea & 4-5 días & \\
\hline Saccharum officinarum & 1 día & \\
\hline Salpichroa origanifolia & 1 día & \\
\hline Tropaeolum majus & 2-5 días & \\
\hline \multicolumn{3}{|l|}{ CLADODIOS } \\
\hline Opuntia spp. & 2-5 días & \\
\hline \multicolumn{3}{|l|}{ FLORES } \\
\hline Eupatorium sp. & $12-24$ horas & \\
\hline Lantana camara & 1-2 días & \\
\hline Thunbergia alata & 1-2 días & \\
\hline Wedelia glauca & 4-5 días & \\
\hline \multicolumn{3}{|l|}{ FRUTOS } \\
\hline Senecio laetevirens (cipsela) & 4-5 días & \\
\hline Solanum melongena (epicarpo) & 5-24 horas & \\
\hline Wedelia glauca (cipsela inmadura) & 4-5 días & \\
\hline \multicolumn{3}{|l|}{ SEMILLAS } \\
\hline Datura ferox (episperma) & 4-24 horas & \\
\hline \multicolumn{3}{|l|}{ CULTIVO IN VITRO } \\
\hline Prosopis alpataco (explante) & 24-36 horas & \\
\hline
\end{tabular}

Para el presente trabajo se ha ensayado la preparación de Gelatina-fucsina y se obtuvieron buenos resultados. La coloración con fucsina básica, es principalmente apropiada para ver tejido vascular. También se pueden utilizar diferentes reactivos que permiten la identificación de sustancias lipofílicas, taninos, almidón, mucílagos, etc.

\section{Resultados}

Se documentan los resultados en la Tabla 1, Fig. 1 y Fig. 2. Por las ventajas que presentó la nueva técnica de clarificación, se generalizó rápidamente su uso, tanto con hojas de diferentes tamaños y consistencias, como diversos órganos vegetales, 
Bol. Soc. Argent. Bot. 53 (4) 2018
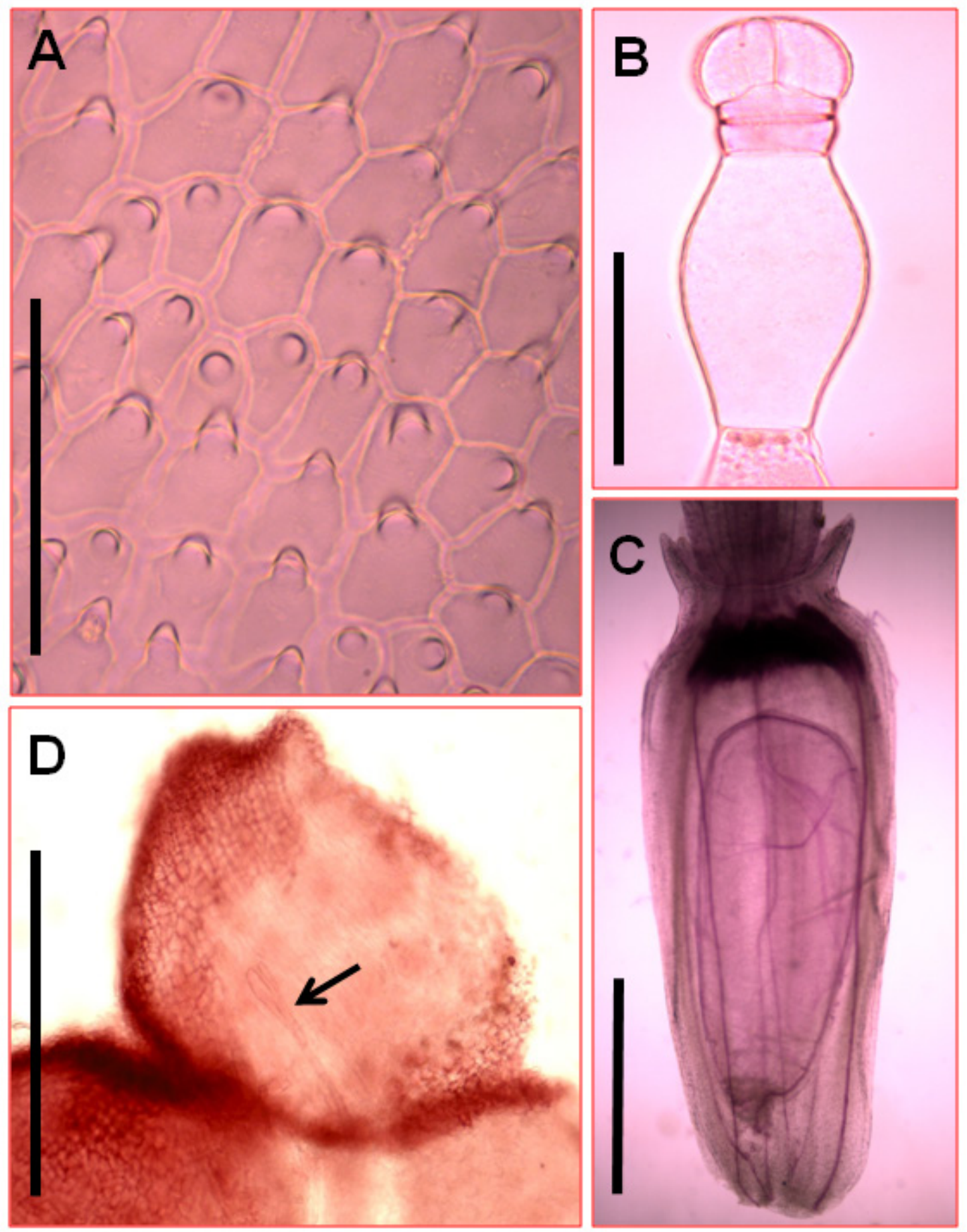

Fig. 2. A-B: Thunbergia alata. A: detalle de las células epidérmicas con papilas excéntricas en los pétalos de la corola. B: porción apical de un pelo glandular presente en la corola. C: Wedelia glauca, fruto tipo cipsela con la semilla, coloreada con fucsina básica, note la vascularización de la semilla y el fruto. D: Prosopis alpataco, explante diafanizado, mostrando la formación de embrión somático con inicio del tejido vascular (flecha). Escalas= A-C: $100 \mu \mathrm{m}$. D: $300 \mu \mathrm{m}$. 


\section{A. M. Arambarri - Nuevo método de clarificación vegetal}

pudiendo regular con facilidad los tiempos del tratamiento. En algunos materiales se encontró que no es necesario practicar el método de clarificación, por ejemplo en raicillas de plántulas incoloras, o las hojas de lechuga (Lactuca sativa) que se decoloraron rápidamente con solución acuosa de hipoclorito de sodio comercial. Sin embargo, en ambos casos se mejoró la transparencia tratando el material con hidrato de cloral como mínimo 24 horas.

\section{Conclusión}

La "técnica de clarificación 5-5-5" se desarrolla a temperatura ambiente, es un método sencillo, económico, práctico y seguro, permite trabajar diferentes órganos y tejidos, tanto de material fresco como de herbario, en este último caso previa hidratación. Es altamente recomendable para investigaciones de materiales delicados (tricomas, flores, explante) y aquellos que requieren mantener una orientación para su análisis, en especial cuando son de reducido tamaño (foliólulos).

El método muestra las siguientes ventajas, ya que permite:

- realizar clarificación de diferentes órganos o parte de los mismos.

- diafanizar materiales muy delicados, como explantes y flores.

- trabajar con material fresco como tejidos secos o de herbario, previa hidratación.

- mantener la orientación del material.

- controlar el proceso de clarificación.

- lograr una perfecta clarificación.

No es un método agresivo porque no requiere de calentamiento de los compuestos actuantes.

La única desventaja que se detectó, es que el tratamiento no es inmediato en la mayoría de los materiales vegetales utilizados.

\section{Agradecimientos}

A mis colegas que han contribuido con el uso, ensayo, comentarios y/o preparados para las ilustraciones de esta técnica, Silvana Auguet, Patricia Boeri, Marta Colares, Marcelo P. Hernández, Santiago M. Martínez Alonso, M. Cecilia Novoa, Leandro Nughes, Lucía Valerga. Mi especial agradecimiento a Vanesa G. Perrotta por su colaboración en la preparación de la Figura 1. Lo hago extensivo a los revisores y en especial a la editora Ana M. González por sus valiosos comentarios y sugerencias.

\section{Bibliografía}

ALTAMIRANO, C. G. \& M. E. YAJÍA. 2017. Improntas reveladora de caracteres micrográficos epidérmicos con aplicación en el control de calidad de plantas medicinales. Dominguezia 33: 33-36.

ARAMBARRI, A. M., M. C. NOVOA, N. D. BAYÓN, M. P. HERNÁNDEZ, M. N. COLARES \& C. MONTI. 2011. Anatomía foliar de arbustos y árboles medicinales de la región chaqueña semiárida de la Argentina. Dominguezia 27: 5-24.

ARAMBARRI, A. M., C. MONTI, N. D. BAYÓN, M. P. HERNÁNDEZ, M. C. NOVOA \& M. COLARES. 2012. Ecoanatomía foliar de arbustos y árboles del distrito chaqueño oriental de la Argentina. Bonplandia 21: 5-26.

BORELLI, L. \& A. PELLIZA DE SBRILLER. 2014. Determinación de la composición botánica de la dieta de herbívoros a través de la técnica microhistológica. En: ZARLAVSKY G. E. (ed.). Histología vegetal: técnicas simples y complejas, pp. 153-161. Sociedad Argentina de Botánica, Buenos Aires, Argentina.

CASTEllaro, G. G., N. F. SQUella, R. T. ULLRICH, C. F. LEÓN \& S. A. RAGGI. 2007. Algunas técnicas microhistológicas utilizadas en la determinación de la composición botánica de dietas de herbívoros. Agric. Téc. 67: 68-93.

CID, M. S. \& P. SIERRA. 2004. Identificación de plantas tóxicas en el contenido gastrointestinal de rumiantes [online]. Disponible en: www.e-campo. com. [Acceso: 18 mayo 2018].

DIZEO DE STRITTMATTER, C. 1973. Nueva técnica de diafanización. Bol. Soc. Argent. Bot. 15: 126129.

FOSTER, A. S. 1949. Practical plant anatomy, 2nd ed. D. van Nostrand Co., New York, USA.

FREIRE, S. E., A. M. ARAMBARRI, N. D. BAYÓN, G. SANCHO, E. URTUBEY, C. MONTI, M. C. NOVOA \& M. N. COLARES 2005. Epidermal characteristics of toxic plants for cattle from the Salado river basin (Buenos Aires, Argentina). Bol. Soc. Argent. Bot. 40: 241-281.

GATTUSO, M. 2013. Micrografía analítica y la farmacobotánica. Dominguezia 29: 5-9.

HERNÁNDEZ, M. P., A. M. ARAMBARRI \& L. NUGHES. 2016. Efectos de la contaminación ambiental sobre la anatomía de la hoja de Celtis ehrenbergiana (Celtidaceae) en la ciudad de La Plata (Argentina). Yvyraretá 23: 30-40. 
HERR, Jr., J. M. 1971. A new clearing-squash technique for the study of ovule development in angiosperm. Amer. J. Bot. 58: 785-790.

JOHANSEN, D. A. 1940. Plant microtechnique. McGraw-Hill Book Co., New York, New York, USA.

NUGHES, L., M. COLARES, M. P. HERNÁNDEZ \& A. M. ARAMBARRI. 2013. Morfo-anatomía de las hojas de Celtis ehrenbergiana (Celtidaceae) desarrolladas bajo condiciones naturales de sol y sombra. Bonplandia 22: 47-58.

PAYNE, W. W. 1969. A quick method for clearing leaves. Ward's Bulletin 8: 4-5.

VASCO, A., M. THADEO, M. CONOVER \& D. C. DALY. 2014. Preparation of samples for leaf architecture studies, a method for mounting cleared leaves. Appl. Plant Sci. 2: 1-4 [online]. Disponible en: http://www.bioone.org/loi/apps [Acceso: 20 mayo 2018].
YAGUEDDÚ, C. \& M. S. CID. 1992. Caracteres epidérmicos de Dicotiledóneas de la Pampa deprimida Bonaerense, de utilidad en microanálisis de dietas. Rev. Arg. Prod. Anim. 12: 265-279.

ZARLAVSKY, G. E. 2014. Histología vegetal: técnicas simples y complejas. Sociedad Argentina de Botánica, Buenos Aires. Argentina.

Recibido el 21 de junio de 2018, aceptado el 14 de agosto de 2018. Editora: Ana M. Gonzalez. 